\title{
Ideal Corporal Masculino: Percepciones De Estudiantes Universitarios De Nutrición
}

\author{
María Leticia Bautista-Díaz (PhD) \\ Ana Karen Galván-Juárez. \\ Itzel Esmeralda Martínez-Fernández \\ Alan Javin Álvarez-Ríos \\ Rubén García-Cruz (Ed.D) \\ Universidad Autónoma del Estado de Hidalgo/ \\ Instituto de Ciencias de la Salud. Hidalgo, México
}

doi: 10.19044/esj.2017.v13n26p1 URL:http://dx.doi.org/10.19044/esj.2017.v13n26p1

\begin{abstract}
Body image is a dynamic entity that includes three components: perceptive, cognitive-affective and behavioral; It is constructed of characteristics such as age, sex and educational level, as well as the ideas established by the context. The interaction between the psychological and the social can lead to the development of clinically relevant behaviors. Thus, the objective of the present investigation was to know the perceptions on the male body ideal of the college students of nutrition. Through a focus group, since the qualitative-phenomenological approach and based on the participants discourse, four categories of analysis were derived. It was found that media (including social networks), cultures, stereotypes and beliefs are predominant factors in the construction of the male body ideal, which is reflected in self-esteem, health, economic, occupational or professional opportunities, and to achieve it, can be developed eating disorders symptoms or muscular dimorphic disorder symptoms. It is conclude that the focus group allows knowing the perceptions related to male body ideal by college students of nutrition, where it was showed that, also the society pressures to achieve that ideal, for example, participants express body satisfaction, but wish to modify their composition, so their beliefs determine what must be done to reach such ideal. Therefore, it is evident the importance of carrying out intervention programs at the primary level in college students, from health psychology.
\end{abstract}

Keywords: Male body ideal, social-cultural factors, nutrition 


\section{Resumen}

La imagen corporal es un entidad dinámica que incluye tres componentes: perceptivo, cognitivo-afectivo y conductual; se construye a partir de características como edad, sexo y nivel educativo, así como de las ideas establecidas por el contexto. La interacción entre lo psicológico y lo social, puede conducir al desarrollo de comportamiento clínicamente relevante. Así, el objetivo de la presente investigación fue conocer las percepciones del ideal corporal masculino de universitarios de la licenciatura en nutrición. A través de un grupo focal, desde el enfoque cualitativofenomenológico y con base al discurso de los participantes, se derivaron cuatro categorías de análisis. Se encontró que medios de comunicación (incluidas las redes sociales), cultura, estereotipos y creencias, son factores predominantes en la construcción del ideal corporal masculino, mismo que se refleja en la autoestima, salud, oportunidad económica, laboral o profesional, y para alcanzarlo se puede desarrollar sintomatología de trastornos de la conducta alimentaria o de trastorno dismorfico muscular. Se concluye que el grupo focal permite conocer las percepciones relacionadas con el ideal corporal masculino de universitarios de la licenciatura en nutrición, donde se revela que la sociedad también ejerce presión para alcanzar dicho ideal, por ejemplo, los participantes expresan satisfacción corporal pero desean modificar su composición, así sus creencias determinan lo que se debe hacer para ajustarse a dicho ideal. Por tanto, se evidencia la importancia de llevar a cabo programas de intervención a nivel primario en universitarios, desde la psicología de la salud.

Palabras-claves: Ideal corporal masculino, factores socioculturales, nutrición

\section{Introducción}

La imagen corporal es la representación mental del cuerpo que cada persona construye, así, necesariamente no tiene que ver con la composición física real del cuerpo, si no con las ideas, sentimientos y actitudes que se generan hacia el propio cuerpo (Fisher, 1986; Raich, 2004; Salaberria, Rodríguez, \& Cruz, 2007; Vaquero-Cristobal, Alacid, Muyor, \& LópezMiñarro, 2013).

Diversos especialistas sostienen que la imagen corporal contempla tres componentes: 1) perceptivo, que son imágenes del tamaño y la forma del cuerpo o de algunas partes de este; 2) cognitivo-afectivo, el cual señala la valoración (actitudes y sentimientos) del cuerpo o algunas de sus partes, a través de autoafirmaciones y creencias asociadas al mismo e involucra experiencias de placer/displacer, satisfacción/insatisfacción, ligados a la apariencia corporal externa; 3) conductual, que son las acciones dirigidas a 
afirmar, alcanzar o rechazar la representación mental, mismas que están estrechamente relacionadas con los dos componentes anteriores (Cash, 1990; Cruz, Maganto, \& Echebarría, 2002; Raich, 2004, 2016; Raich, SánchezCarracedo, \& López, 2008; Thompson \& Heinberg, 1999). Por ello, se ha propuesto que la imagen corporal es una entidad multidimensional compleja y dinámica, que varía en función de las experiencias internas (psicológicas) y externas (medio ambientales; Baile, 2003).

En el caso de las experiencias medioambientales, se ubica la influencia del contexto sobre la percepción de la imagen corporal, y esta no es reciente, ya que se ha manifestado a lo largo de la historia de la humanidad. Al respecto, Gómez (2014) explica que desde diferentes sociedades y culturas, la representación de la feminidad se asocia con la sumisión, abnegación, dependencia y expresividad, en tanto, que la masculinidad se relaciona con el poder, agresividad, fuerza y la libertad. Por tanto, al hombre se le "enseña" que, músculo es igual a fuerza y ésta es lo mismo que hombría y virilidad.

Actualmente, el desarrollo tecnológico en los medios de comunicación, propicia la difusión de mensajes de forma rápida $y$ globalizada, la publicidad está cargada de mensajes para representar -en las mujeres- un ideal estético de delgadez, mientras que en los hombres, los mensajes se dirigen hacia una figura del varón musculoso - pero sin grasa-, en muchos casos, ambos ideales son inalcanzables, lo que puede conducir al desarrollo de comportamientos alterados en un afán de conseguir dicho ideal. Al respecto, Thompson y Heinberg (1999), Thompson y Smolak, (2001), Thompson, Schaefer y Menzel (2012) y Raich et al. (2008), sostienen que los medios de comunicación juegan un papel importante en el desarrollo de alteraciones de la percepción de la imagen corporal o de comportamiento alimentario de riesgo, que pueden conducir a trastornos de la conducta alimentaria (TCA) o a trastornos de la imagen corporal, como es el trastorno dismórfico muscular. Así, el estándar social de la delgadez para las mujeres o del cuerpo musculoso para los hombres, se ha convertido en símbolo asociado al éxito y a la independencia (Ianotti, 2005).

Como se refirió previamente, en los varones el estereotipo culturalmente establecido es el ideal de un cuerpo mesomorfo, es decir con musculo pero sin grasa, en tanto que para las mujeres es ectomórfo (delgado y estilizado), por lo que hay cierta presión para lograr un cuerpo con dicha condición, así las acciones a seguir en búsqueda de dicho ideal es la realización de ejercicio físico excesivo, dietas restrictivas, consumo de fármacos o sustancias como los esteroides anabolizantes, entre otras, para obtener una figura que represente -en los hombres fuerza y poder-y para las mujeres, un figura que muestre belleza y éxito (Fanjul \& González, 2011; Raich, 2004). Así, cada vez más mujeres muestran preocupación por la 
imagen corporal aun teniendo un peso normal, ya que perciben ciertas partes (e.g., brazos, cintura, vientre, piernas, etc.) del cuerpo, más grandes de los que en realidad son. Diferencialmente, los hombres muestran preocupación por el tono muscular bien definido de los muslos, piernas, hombros y pectoral. Respecto al volumen de este último, se ha referido que un hombre con un pectoral pequeño o sin volumen, se percibe como poco masculino (Raich, 2004).

Sin embargo, la percepción de la imagen corporal de dos hombres con misma altura y mismo peso, puede ser totalmente diferente, debido a sus características y experiencias personales. Bobadilla y López (2014) plantean que la discrepancia entre la imagen formada y la composición real, puede determinar acciones extremas a seguir, no sólo entre las mujeres, ya que actualmente existe mayor presencia de dichas conductas entre los hombres, dichas conductas los pude conducir al exceso de peso debido al desequilibrio que ocasionan las dietas inadecuadas, el ayuno o ejercicio en exceso. De manera general, la insatisfacción corporal es una autoevaluación corporal negativa, se define como un grado elevado de malestar con la figura corporal que altera la vida cotidiana de quien la padece y la conduce a la realización de comportamientos que atenta contra la salud física y psicológica (Raich, 2016).

Gómez, Sánchez y Navarrete (2012) encontraron que en una muestra de estudiantes de secundaria y preparatoria (12-19 años), los hombres se encuentran más insatisfechos con su apariencia física y presentan mayor distorsión de su imagen en comparación con las mujeres. Contrariamente, Bobadilla y López en 2014, hallaron que hombres y mujeres (con rango de edad entre 15 y 69 años) reportaron índices elevados de distorsión corporal, no obstante, las mujeres están más insatisfechas con su cuerpo y son más susceptibles al ideal de delgadez que los hombres. En estudiantes de universitarios de nuevo ingreso de una universidad de Chile, se encontró que un mayor porcentaje de mujeres en comparación de los hombres presentan insatisfacción corporal (Lagos, Quilodrán, \& Viñuela, 2012). Mientras que en México, se encontró que hombres y mujeres presentan el mismo nivel de insatisfacción corporal (Unikel, Díaz de León, \& Rivera, 2016). Al respecto, Vaquero et al. (2013) señalan que una mayor influencia sociocultural del modelo de delgadez se asocia con una mayor percepción de la grasa corporal, con mayor insatisfacción corporal y por tanto, a una menor valoración del autoconcepto físico general. Los autores concluyen, que estás alteraciones, conducen a adolescentes y jóvenes a desarrollar un TCA.

Aunque son escasos los estudios de prevalencia de TCA en México, se evidencia que aunque en mayor medida las mujeres son víctimas de los TCA, el número de varones con esta psicopatología, ha aumentado en forma constante en los últimos años (Medina-Mora, et al., 2003; Unikel-Santoncini, 
Bojórquez-Chapela, Villatoro-Velázquez, Fleiz-Bautista \& Medina-Mora, 2006; Unikel, Villatoro, Medina-Mora, Fleiz, Alcántar, \& Hernández, 2000). Al respecto, Moreno y Ortiz (2009) encontraron que estudiantes (hombres y mujeres) de secundaria, presentan preocupación por su imagen corporal, niveles medios-bajos de autoestima, y que aquellos con insatisfacción corporal presentan mayor tendencia hacia los síntomas de TCA. Alfonso, Walters y Sánchez (2012) identificaron en una muestra de atletas varones universitarios que $76 \%$ se adhiere de manera moderada o alta al modelo tradicional de la masculinidad, $15 \%$ reportan conducta alimentaria de riesgo, mientras que $9 \%$, refieren tener insatisfacción con su figura corporal.

La carrera profesional de nutrición, es una disciplina que se encarga de estudiar la alimentación-nutrición humana y su relación con los procesos biológicos, químicos y metabólicos para preservar la salud física (Otero, 2012). En un estudio realizado por López et al. (2008) con una muestra de estudiantes pertenecientes a un centro universitario de ciencias de la salud, entre ellas, la de nutrición, se encontró que los alumnos de dichas disciplinas reportan insatisfacción corporal, así como baja autoestima. Recientemente, se encontró que estudiar una carrera de ciencias biológicas de la salud representa en los varones un factor importante de riesgo para desarrollar sítomas de TCA (Unikel et al., 2016).

A manera de síntesis, las alteraciones con la imagen corporal no son exclusivas de las mujeres, ya que, actualmente se ha observado una mayor presencia entre los hombres. Dichas alteraciones se presentan desde la etapa de la niñez hasta la adultez temprana, concretamente, en esta última etapa del desarrollo los estudiantes de nutrición, serán profesionales de la salud que atenderán problemáticas relacionadas con la alimentación y nutrición humana, y ellos mismos pueden estar inmersos, -entre otros aspectos relacionados con la inserción a la vida universitaria-, en la presión social para alcanzar dicho ideal y llevar a cabo ciertos hábitos considerados como no saludables, aunado a que la mayoría de las investigaciones al respecto son de corte cuantitativo, como se demostró en una revisión de la literatura (Mendieta-Izquierdo, 2014). Aun con lo valioso de los estudios cuantitativos, todavía resulta limitado el conocimiento a profundidad de las experiencias de los varones sobre su imagen corporal, por lo que, resulta relevante conocer la perspectiva respecto a la imagen corporal, de esta población en específico. Así, el objetivo de la presente investigación fue conocer las percepciones del ideal corporal masculino de universitarios de la carrera de nutrición.

\section{Método}

\section{Diseño de investigación}

Observacional, transversal de tipo cualitativo (fenomenológico), una de las principales características de este enfoque de investigación, es que se 
posiciona en el nivel de descripción, interpretación y entendimiento de la realidad de los participantes, esto a través de sus experiencias, opiniones o perspectiva respecto a un tema o una situación en específico (ÁlvarezGayou, 2003; Denzin \& Lincoln, 2012; Dongre, Deshmukh, Kalaiselvan, \& Upadhyaya, 2009; Kerlinger \& Lee, 2002; Silverman, 2016). El grupo focal es un medio idóneo para recabar datos desde este enfoque, ya que, permite un desarrollo natural de los sucesos y al mismo tiempo, se realiza una construcción dinámica de la situación (Alvarez-Gayou, 2003; Hamui-Sutton \& Varela-Ruiz, 2013).

\section{Participantes}

Seis varones estudiantes de la carrera en Nutrición, pertenecientes a una universidad pública ubicada en el Estado de Hidalgo, México, con edad entre 19 y 20 años, solteros, inscritos en el primer semestre de la carrera de Nutrición, sólo uno de ellos es usuario de gimnasio. Álvarez-Gayou, 2003 y Denzin y Lincoln, 2012, sostienen que el tamaño adecuado para la realización de un grupo focal debe ser entre seis y diez participantes, ya que no se pretende la representatividad, ni la transferibilidad, aunado a que un grupo pequeño permite que "todos" los participantes realicen autoexpresiones (confesiones) de forma natural.

\section{Escenario}

El trabajo de campo se llevó a cabo en un aula dentro de las instalaciones de la institución educativa. El aula es un espacio amplio (6m $\mathrm{x}$ $5 \mathrm{~m}$, aproximadamente), bien iluminado y ventilado, permitió colocar los pupitres en forma de "O" para garantizar la no jerarquía y la apertura de la conversación así como la adecuada proyección del estímulo (video).

\section{Medio de recolección de datos}

La técnica del grupo focal se contempla como una entrevista grupal, más que individual. El análisis y comprensión de los datos se basó en el enfoque fenomenológico, ya que, permite entender el significado de las experiencias y los actos de quienes las comparten (Ayala, 2008). Para desarrollar la discusión focalizada respecto a la imagen corporal, se utilizó como estímulo, el video "Estereotipo de belleza masculina" que se encuentra de acceso libre en la Web en la siguiente dirección https://www.youtube.com/watch?v=1YuHJmIHzE8, el cual tiene una duración de 1.34 minutos. Previamente, en consenso entre los investigadores se diseñó un guión de cinco preguntas todas sobre la percepción de la imagen corporal. Al término del la proyección del video, se les cuestionó a los participantes con la primera pregunta de dicho guion: ¿Ustedes que opinan respecto a la imagen corporal de los hombres? Posteriormente, durante el 
transcurso de la discusión fue necesario realizar también la siguiente pregunta -incluida en el guion- ¿Cómo debe ser el cuerpo masculino?

\section{Aparatos}

Una Lap top de la marca Dell Inspiron 14, serie 3000 de 14-inch.

\section{Procedimiento}

Identificados los participantes, de manera individual se les explicó e invitó a colaborar en el estudio. Posteriormente, firmaron el consentimiento informado, se acordó lugar, fecha y hora para la realización de la investigación. Se destaca que en el caso de las investigaciones con mínimo riesgo (como es el caso de la presente investigación), no es requisito someter el protocolo a Comité de Ética de la institución educativa, no obtante, se toma en cuenta el Código Ético del Psicólogo para realizar investigaciones con seres humanos (Sociedad Méxicana de Psicología, 2010). Uno de los autores del presente trabajo fungió como moderador, quien proporcionó las pautas del grupo focal, incluida la solicitud de autorización de la grabación y los fines de los datos. Otro de los investigadores, fue el observador/relator del mismo.

\section{Análisis de los datos}

Con los datos recabados y con el análisis directo del audio se realizó una codificación abierta (por pares entre los investigadores) y posteriormente, se derivaron cuatro categorías.

\section{Resultados}

El grupo focal se llevó a cabo el día y hora planeados, los seis participantes llegaron puntuales a la cita. Posterior a la proyección del video y lanzar la pregunta estímulo, los participantes guardaron silencio unos segundos, de manera espontánea uno de los ello dio su opinión, los demás escucharon atentos y se desencadenó la discusión, misma que fue respetuosa aún con opiniones diferentes. La participación fue continua, aunque a veces fue de mayor frecuencia en algunos de los estudiantes. Los gestos y posturas de los participantes en general, revelaron su agrado con la dinámica y la temática.

Así, para alcanzar el objetivo de la investigación que fue conocer las percepciones del ideal corporal masculino de universitarios de la carrera de nutrición, se analizaron los datos obtenidos durante el grupo focal $\mathrm{y}$ posteriormente, se derivaron cuatro categorías: 1) Medios de comunicación y sociedad; 2) Cultura, creencias y estereotipos corporales; 3) Composición corporal y autovalía; 4) Cuerpo, salud: oportunidad profesional y laboral. A 
continuación se describen fragmentos del discurso textual y su interpretación.

\section{Medios de comunicación y sociedad}

"Con todo lo que has visto en la televisión de chiquito, lo que te dicen tus papás, lo que te dicen tus amigos...la música que te gusta, los programas, con todo eso, formas una imagen para llegar a ser según, lo que tú quieres...", "Los géneros de música, la publicidad de comerciales...siempre pasan a tipos, así como musculosos para todo", "Pues sí influyen bastante, porque a lo mejor no todos, pero alugnos dicen, no pues, yo quiero estar como el cantante, o tal luchador o tal personaje... pero si tratan de verse o parecerse a ese tipo...", "Las personas ven en la televisión a alguien musculoso... van a querer ser como él...es lo que te dice la sociedad". "...mis compañeros, maestros incluso las mismas niñas, me decían ya trabájale [realizar acciones para modificar la forma del cuerpo]...desde ahi, decidí subir más de peso porque estoy flaco”, "Depende dónde creces, con quién te juntas por ejemplo, si piensas en la imagen de alguien...que le guste el gym [gimnasio] y sus amigos, probablemente estén como más trabados [corpulentos], más marcados [que se note la figura de los músculos] pues vas a pensar que la imagen, es ese fitness [figura corporal]", "Ya no ves como mucha tele, más bien entras a Instagram ...todo mundo es modelo en Instagram, tú ves perfiles y es lo mismo, chavas, siempre es lo mismo".

Con este discurso, es claro observar y se confirma el papel que juegan los medios de comunicación y la sociedad en general en la construcción del ideal corporal; en el caso de la familia y de los pares en particular, se refirió que influyen en las percepciones de las personas para construir un cierto modelo estético corporal, mismo que se internaliza y se convierte en un guía a seguir, al respecto, se declaró que desde etapas tempranas del desarrollo, tal es el caso de la niñez, dicho ideal corporal afecta los pensamientos, sentimientos y conductas cotidianas que prevalecen hasta la adultez, se puntualiza que no se observó crítica hacia los mensajes de los medios, pares o familia, puede ser que sólo se aceptan con naturalidad.

Lo anterior lo confirman Bazán y Miño (2015) y Cash y Pruzinsky (2002), cuando refieren que los medios de comunicación, son voceros y constructores de expectativas sociales y tienen un rol fundamental en la expansión del modelo estético, ya que promueven el ideal corporal a través de la difusión de patrones de belleza, ejerciendo un efecto poderoso de internalización en la población. Asimismo, Thompson y Heinberg (1999) mencionan que los medios de comunicación contribuyen en el desarrollo del trastorno de la imagen corporal y/o del comportamiento alimentario. Con relación a esta vía de comunicación, la tecnología avanza a pasos acelerados, 
hasta hace unas décadas, la televisión era el principal medio de comunicación, hoy en día las redes sociales han ganado el terreno justamente entre la población en riesgo (adolescentes y jóvenes-adultos) para transmitir mensajes y modelos, al respecto, otro aspecto que puede ser positivo o negativo, es que las redes sociales están disponibles en todo momento, a diferencia de la televisión, que era y es necesario un espacio y tiempo específico para su contacto. En muchos casos, cuando se internaliza un modelo estético corporal y se desea alcanzarlo se realizan conductas que atentan contra la salud física y psicológica. En un estudio de corte cuantitativo con estudiantes de la carrera de Nutrición, se encontró que más de un tercio de dicha muestra $(33 \%)$, reportaron tener insatisfacción corporal, internalización del modelo estético corporal y realización de conductas alimentarias de riesgo para desarrollar TCA, como atracones alimentarios, dietas restrictivas y ayunos (Chávez-Rosales, Camacho, Maya, \& Márquez, 2012).

Sin embargo, no solo los medios de comunicación influyen en la construcción de ideales corporales, sino también familia y amigos, como lo expresaron los participantes, en el caso de la familia (padres y hermanos), es la institución que gesta ideas e imágenes y es el primer contacto con la sociedad en general, ya que, tanto la sociedad próxima (amigos y compañeros) como distal (comunidad y sociedad general) es la que refuerza y conduce a la internalización dicho ideal. De este modo, las relaciones sociales de manera general, son un pilar importante, ya que, es la conexión que se establece entre las personas y su cultura. En este caso, se critica la delgadez en los hombres y se les responsabiliza de su condición por lo que se les alienta a trabajar su figura corporal. Así, en cuanto a la imagen corporal, las relaciones de amistad, familiares, incluso laborales, son constructores de un ideal corporal y al mismo tiempo, indican las pautas a seguir, tanto acciones como decisiones para alcanzar dicho ideal.

\section{Cultura, creencias y estereotipos corporales}

"Yo siento que, igual tiene que ver con las creencias... allá en China, es como más de ser delgados, y el que tiene un cuerpo muy grande y voluminoso no es aceptado...tiene que ver la creencia...bueno, su religión no les permite, así como, que comer mucho". "Te dicen, un egipcio y te imaginas como algo, como morenillo, alto, delgado; te imaginas un ruso, muy marcado un tipo como grueso, alto como pechudo y barbón, ósea, como que, sí depende de dónde vivimos". "Cada país tiene como distintivo el ideal, estos distintivos marcan las creencias...nosotros nos vamos a fijar precisamente de nuestro país". "Así como pues, de los mexicanos, que se distinga... como chaparro y panzón, no...pero como que lo que todos buscan, es un cuerpo trabajado [desarrollado en el gimnasio, no de manera 
natural], pero igual, no es tan fácil...lo más fácil es estar panzón”. "Yo creo que va cambiando con el tiempo... yo creo que como en los años 50 y 80 podríamos decir que era como del machote, como panzón...no era un cuerpo como muy marcado, muy estético, ahora la figura está más influenciada por la globalización", "Muchos piensan que estar flaco es bueno y gordo malo, o cosas así", "Ya todos quieren estar más fuertes, más fornidos", "En México...siempre cuando ven a alguien trabajado, lo critican mucho".

Respecto a esta categoría de análisis, Camarena y Tunal (2009) indican que las creencias y persepciones son dimensiones de la cultura, éstas forman parte de la esfera de la vida social, por tanto, son constructoras de los ideales y así, es como se logra una cohesión social a través de una configuración colectiva. González (1999) sostiene que las creencias de un grupo social, representan un factor predominante en la opinión de la sociedad, ya que, influyen en las percepciones de sus miembros.

Por tanto, los estereotipos surgen de ciertos contextos y situaciones en los que se está inmerso, así la población organiza las creencias (estereotipos), las generaliza y al mismo tiempo da las pautas para alcanzar cierto ideal, en ese caso de composición corporal. No obstante, entre la población mexicana, la globalización, ha llevado a vivir una situación compleja, ya que por un lado, se busca un ideal y por el otro, se vive en un ambiente obesogénico, el cual fomenta el consumo de comida con alto contenido calórico y bajo valor nutricional, así como un alto sedentarismo, que en interacción conducen al exceso de peso (Múñoz-Cano, CórdovaHernández, \& Boldo-León, 2012); es por ello, que los estudiantes de nutrición refieren que actualmente es más fácil acumular grasa abdominal. Sin embargo, hasta hace tres décadas, la representación de la figura corporal del mexicano de los mismos mexicanos, era la de una figura hectomorfa, no obstante, más recientemente, se le brinda mayor importancia al tener un tipo de cuerpo mesoformo (musculoso y sin grasa) en los varones, pero contradictoriamente, también se critica a quienes tienen una forma corporal considerada no natural (trabajada en un gimnasio), considerándolo fuera de la norma y por realizar acciones que lo conducen a una figura con músculos definidos. Así, las creencias y la sociedad generan los estereotipos y por ende, propician acciones para lograr un acercamiento a ciertos ideales.

\section{Composición corporal y autovalía}

"No requiero de una parte fisica perfecta, finalmente me siento cómodo con mi cuerpo, y sí, quisiera hacer cambios en mi cuerpo, pero no es algo que me obsesione...", "Me siento cómodo, pero aún, no estoy como quiero", "Me sentiría más seguro en algunas ocasiones, más seguro para ciertas cosas, no es que quiera decir, que no me sienta seguro ahora... yo siempre he sido así, como muy tímido para las chavas, siento que eso sería 
lo único que se me facilitaría", "Tendrías más chicas, más atención y eso". "No creo que la forma física cambie la forma en que eres tú...de manera fisica si cambias, pero son cambios muy superficiales". "Ya cuando llegas a tu objetivo [una forma corporal deseada], sientes que puedes llegar a ser más liberal, te sientes más cómodo contigo mismo...eres como más abierto...puedes llegar y hablarle a cualquier persona", "Quisiera aumentar músculo y volumen", "Hay puntos que digo, me gustaría tener más musculo, los pectorales abiertos", "Yo igual siento que es como motivación, por ejemplo, yo como cuando entré al gimnasio, mi objetivo sólo era estar marcado, bueno no sólo por estarlo, sino por estar bien, estar ahí [refiriéndose al gimnasio construyendo su cuerpo]", "No es que me sienta mal [refiriéndose a su cuerpo] pero no me gusta cómo me veo en el espejo...", "Yo como soy muy indeciso, cuando llegue a ese objetivo [una figura musculosa] buscaría todavía más [continuar trabajando la forma corporal]... si llegó a eso me sentiría muy bien", "No quiere decir que lo que veas, lo desees, sino que lo veas y se puede ver bien, y si en ese momento te va a ser feliz y te va a llenar, pues está chido [expresión de estar bien] y es algo que tú quieres, no algo que te imponen", "Menos panza y más marcado", "Aumentar un poco más mi índice de masa corporal", "Yo llegue a ser de esos tipos que criticaban a los que estaban marcados [músculos definidos], decía ;da asco mucho músculo!, ver proteínas, creía que eran esteroides, conforme fui pasando el tiempo en el gimnasio, en vez de asco sentí admiración... mi objetivo cambió", "Si, ósea, si van al gym [gimnasio] sientes como, ...quiero estar, así como súper musculoso" ¿Qué le metes? le meto prote [proteínas] y chochos [medicamentos homeopáticos] y como un buen, o si quiero estar fitness pues le meto menos carne, más trabajo de pesas o más cardio [trabajo cardiovascular]", "Yo creo, que igual muchas veces hay conductas malas, o bueno no tan malas, no indicadas que te llevaron a tener una buena conducta o buen hábito, o bueno en mi caso, para mí es muy adoptado hacer ejercicio, comer bien, ósea, dejarlo todo, fiesta y meterte lo que pudieras y pasarla bien entre comillas y aparte igual, como pasa el tiempo y pasas los años, yo creo que te das cuenta que eso, no esta tan chido [adecuado], al menos no llevarlo siempre, en mi caso fue eso, que tener conductas malas me ayudaron a darme cuenta que eso no está chido [bien]", "Siempre suena a cliché, pero la globalización nos ha pegado a todos", "Somos seres cambiantes, finalmente, lo que hoy nos gusta, mañana nos puede disgustar.

Aun cuando el discurso expresado apunta a la satisfacción de la composición corporal, la mayoría de los participantes desean alcanzar un figura corpulenta y con músculos definidos, ya que ésta, dará seguridad, confianza incluso permitirá el desarrollo de relaciones no sólo sociales en general, sino de tipo interpersonal de pareja, además que poseer una figura 
acorde al ideal que prevalece, permitirá ser centro de atención de los otros y del sexo opuesto, además de que se logrará aceptación social. Aunque se critique un cuerpo trabajado en el gimnasio, también se desea llegar a ser como aquello que se critica y se busca la situación para realizar acciones y alcanzar el ideal. Lo anterior permite observar que se lucha (con pensamientos, intenciones y acciones) por alcanzar un ideal cultural internalizado, que puede responder a necesidades personales internas que llevarían a la felicidad, así el deseo es interno pero se base en lo externo. Por tanto, la percepción e internalización de una imagen corporal puede tener un efecto importante sobre el autoconcepto y autovalía propia -y de los otros-.

Al respecto, se ha encontrado que los hombres realizan acciones para modificar su composición corporal, para evitar burlas de sus pares, ya sea por bajo peso o por exceso de este (Bottamini \& Ste-Marie (2006). El discurso de los participantes, está en línea con lo que se ha postulado teóricamente, ya que, alcanzar un ideal corporal, incrementa la autoestima y se logra la sensación de bienestar físico y emocional, por tanto, la motivación también juega un papel fundamental para realzar ciertas conductas. Al respecto, Ballesteros (2015) sostiene que el deseo que activa y dirige el comportamiento relacionado con la imagen corporal, funge como el impulsor para llevar a cabo ciertas conductas. De este modo, con estos hallazgos se evidencia que también los hombres reciben presión para ajustarse a un ideal corporal, como se hace con las mujeres. Aunado a que con este grupo focal se da cuenta de que los hombres también experimentan insatisfacción corporal, así el peso corporal afecta el nivel de satisfacción corporal y conduce a realizar conductas que pueden afectar la salud física y psicológica, Barrientos, Escoto, Bosques, Enríquez y Juárez (2014) encontraron que hasta $16 \%$ de mujeres y hombres que asisten al gimnasio y hasta $13 \%$ de hombres y mujeres quienes no asisten, reportaron insatisfacción corporal clínicamente relevante; esta se ha definido como el grado elevado de malestar que afecta la vida cotidiana y genera un desequilibrio en la salud mental, en el caso de los varones, la insatisfacción, es por tener una figura corporal delgada y sin músculos definidos. Para ejemplificar lo anterior, casi $40 \%$ de los hombres que acuden al gimnasio presentan síntomas del trastorno dismórfico muscular, como es la preocupación constante de aumentar musculatura, consumo de sustancias (esteroides anabolizantes) y adherencia patológica (dependencia) al entrenamiento, mismos que los lleva a abandonar actividades sociales, laborales e incluso de recreación (Barrientos et al., 2014). Lo anterior contradice lo que se había postulado con relación a que el cuerpo en los varones es menos importante que en las mujeres, ya que, ellos centran su valía en las habilidades verbales o deportivas, sin embargo, se ha demostrado que jerárquicamente, lo que es más importante para los hombres, en primer lugar, es el peso corporal y la musculatura, seguido de una 
cabellera abundante, la estatura y finalmente, el vello corporal generalizado (Tiggemann, Martins \& Churchett, 2008).

\section{Cuerpo y salud: oportunidad profesional/laboral}

"Si quieres estar sano, es hacer ejercicio, comer bien, con disciplina", "He conocido a varios que hacen ejercicio y tienen un cuerpo muscular y sano", "Comer saludable, dormir mejor, dejar el alcohol, un cuerpo sano es aquel, que hace ejercicio, dieta, que no come comida chatarra, azúcares y así", "Muchas personas abusan de las pastillas y no llegan a tener un cuerpo sano”, "El estar marcado [músculos definidos], no quiere decir que estés saludable, un cuerpo sano es el que hace ejercicio, pero creo que tiene que ver con el aspecto físico y se puede ver en la piel, el cabello", "... si lo vemos desde el punto de vista nutricional...tengo un beneficio al cambiar mis hábitos y me beneficia porque me voy a ver bien", "Pues te va a beneficiar a tu salud y también económicamente, que es también lo que buscamos [refiriéndose al producto de la profesión]", "Si, tiene como muchas ventajas más, como nosotros que estamos estudiando nutrición", "Será como parte motivacional con el paciente [mostrar cierta figura corporal]", "Antes hacía ejercicio y entonces me sentía bien, no es como un ideal, si no cómo yo me siento bien", "Me va a ayudar a prevenir enfermedades... me va a ayudar a tener mejor metabolismo y también a prevenir muchas enfermedades, si estoy dispuesto a cambiar", "Como te ven, te dan más trabajo... y si me ven mejor, tendría más gente [refiriéndose a pacientes]", "Me conocen más [como profesional] y después tienes más ingresos, más oportunidades de desenvolverte, conocer a otras personas y de crecer [profesionalmente]".

En esta categoría, a nivel salud se refirió que un cuerpo sano no tiene que ver con una forma o figura, sino con las acciones conscientes que se realizan para lograrlo, como es la alimentación balanceada, adecuados hábitos de sueño y la actividad física necesaria; inversamente, el uso de drogas, la ingesta de fármacos o sustancias para construir músculos y alimentación hipercalórica enferma el cuerpo, que se puede ver reflejado en otros elementos del cuerpo, que no tienen nada que ver con la forma corporal. Es probable que, estos pensamientos estén permeados y comprendidos en función del aprendizaje científico que han recibido los estudiantes de nutrición durante el inicio de su formación profesional, ya que el discurso se centra en términos técnicos profesionales de la nutrición. Esto, contradice el postulado de la influencia sociocultural de manera general y coloca en supremacía lo individual -en el caso de estudiantes de la carrera de nutrición-, cuando el ser humano con oportunidad de recibir estudios profesionales, reconoce el papel activo en su propia salud física $\mathrm{y}$ psicológica. Al respecto, Baile (2003) señala que la imagen corporal no es 
estática, ya que, varía a lo largo de la vida y está en función de las experiencias internas y externas.

En cuanto a la dimensión laboral, los participantes consideran que como nutriólogos, es necesario mostrar una figura corporal sana, sin embargo, en todo momento surge el ideal corporal masculino, al respecto, se ha reportado que los hombres con exceso de peso, no deben ser líderes o directores de personal, por tanto, los hombres reciben presión para mostrar un cuerpo que transmita fortaleza, confianza y respeto profesional (Philips \& Drummond, 2001). Así, una figura apegada al ideal corporal conlleva beneficios a nivel profesional, social y al mismo tiempo, proporciona satisfacción y sensación de éxito (Muñoz, 2014).

\section{Conclusion}

La imagen corporal, no sólo en las mujeres sino también en los hombres, es el resultado de la representación interna, mediada por múltiples factores incluido el psicológico: este último, es punto clave para el comportamiento diferencial de persona a persona, el ideal corporal masculino, se va construyendo desde edades tempranas y hasta la adultez, a través de la influencia cultural, la sociedad en general y la familia en particular, así como de los medios masivos de comunicación y, hoy en día de las redes sociales, todo lo anterior en interacción incita a la modificación, estructuración y reestructuración de la imagen corporal. El grupo focal es una vía valiosa de recolección de datos más específicos, en este caso la percepción, experiencias y vivencias sobre el ideal corporal masulino de varones de la carrera de nutrición. Los hallazgos permiten reconocer que se vive en una sociedad globalizada donde mujeres y hombres, niños y adultos, se influyen para generar un estereotipo, por el que los mismos actores luchan por alcanzarlo, impactando en su sistema de creencias, autoestima, motivación personal, oportunidad económica, laboral o profesional. En el caso de universitarios de la licenciatura en nutrición, su discurso es ambivalente, ya que, por un lado, se expresa la satisfacción con la composición corporal real y por el otro, realizan acciones para mejorarla o piensan en llevarlas a cabo para tener mejores oportunidades personales y sociales. Asimismo, no debe perderse de vista que aunque se critica a aquellos cuerpos con músculos definidos y con volumen no natural o poco común, se desea alcanzar dicha composición.

Ya que, poseer un cuerpo idealizado en los varones (i.e., brazos y pectorales con mayor volumen pero sin grasa), potencializa la posibilidad de adquirir beneficios, estos pueden ser estéticos, económicos, sociales (de pareja e interpersonales), psicológicos y hasta para la salud física. Así, el cumplimiento de cierta composición mejorará la autoconfianza, el autoconcepto y la autovalía. 
El posible abordaje de la nutrición de forma científica, lleva a los estudiantes de la carrera de nutrición a reconocer el papel activo para contribuir con el propio organismo para lograr un cuerpo sano a nivel fisiológico y no reducido a una forma o figura corporal. Los estudiantes universitarios en general, son una población en riesgo para desarrollar enfermedades o trastornos, ya que, pueden darse dos situaciones: 1) mayor independencia aún viviendo con los padres; 2) mayor independencia viviendo lejos de la familia, en el segundo caso, ellos asumen su propio estilo de alimentación, por lo que se vuelve una etapa crítica para la salud presente y futura. Ya que, en el caso de los hombres con cuerpo delgado se les exige modificarlo por uno más corpulento, así para cumplir cierta meta, pueden llegar a realizar dietas desequilibradas, realizar ejercicio excesivo o ingerir sustancias nocivas para la salud, que en sus inicios, podrían parecer inofensivas pero pueden convertirse en psicopatologías más complejas, ya que sus efectos no son inmediatos, sino a mediano o largo plazo.

Estos hallazgos permiten observar la importancia de la implementación de programas de intervención o de estrategias desde la psicología de la salud dirigidas a la promoción de la salud relacionadas con la imagen corporal y sus consecuencias, tanto en estudiantes universitarios de la carrera de nutrición en particular, como de las disciplinas relacionadas con la salud, donde una modalidad inter o multidisciplinar mejorará los resultados, ya que son una población en riesgo.

Finalmente, se reconoce como principales limitaciones: 1) contar con uno solo grupo focal; 2) los participantes son del primer semestre de la carrera de nutrición, 3) no se indagó sobre la orientación sexual de los participanes. Por lo que se abre la oportunidad de ampliar la investigación: hasta encontrar saturación de los datos con otros grupos focales y con estudiantes de otras carreras; considerar las vivencias de alumnos de semestres avanzados; incluir la percepción con base a la orientación sexual. Asimismo, sería interesante conocer la percepción de las universitarias sobre los cuerpos masculinos y viceversa, es decir, identificar la percepción de los varones sobre la figura corporal femenina vigente.

\section{References:}

1. Alfonso-Toro, J., Walters-Pacheco, K., \& Sánchez, I. (2012). El cuerpo en forma: masculinidad, imagen corporal y trastornos en la conducta alimentaria de atletas varones universitarios. Acta de Investigación Psicológica, 2, 842-857. Recuperado de http://www.scielo.org.mx/scielo.php?script=sci_arttext\&pid=S200748322012000300007

2. Alvarez-Gayou, J. (2003). Cómo hacer investigación cualitativa: fundamentos y metodología. México: Paidós. 
3. Ayala, R. (2008). La metodología fenomenológico-hermenéutica de M. Van Manen en el campo de la investigación educativa. Posibilidades y primeras experiencias. Revista de Investigación Educativa, 26, 409-430.

4. Baile, J. (2003). ¿Qué es la imagen corporal? Cuadernos del Marqués de San Adrián, 2,53-70.

5. Ballesteros, M. (2015). Motivación: perspectivas teóricas y consideraciones. Revista Educación, 33, 153-170.

6. Barrientos, N., Escoto, C., Bosques, L.E., Enríquez, J. \& Juárez, C. S., (2014). Interiorización de ideales estéticos y preocupación corporal en hombres y mujeres usuarios de gimnasio. Revista Mexicana de Trastornos Alimentarios, 5, 29-38.

7. Bazan, C., \& Miño, R. (2015). La imagen corporal en los medios de comunicación masiva. Psicodebate, 15(11), 23-47.

8. Bobadilla, S., \& López, A. (2014). Distorsión de imagen corporal percibida e imaginada. Revista Médica del Instituto Mexicano del Seguro Social, 4, 408-414.

9. Bottamini, G., \& Ste-Marie, D. M. (2006). Male voices on body image. Journal of Men's Health, 5(2), 109-132.

10. Camarena, M., \& Tunal, G. (2009). La religión como una dimensión de la cultura. Nómadas, 22, 1-15.

11. Cash, T. F. (1990). Body image enhancement: A program for overcoming a negative body image. New York: Guilford.

12. Cash, T. F., \& Pruzinsky, T. (2002). Body images: A handbook of theory, research, and clinical practice. New York: Guilford Press.

13. Cruz, S., Maganto, C., \& Echebarría, A. (2002). Investigaciones en Psicología Clínica. España: Ibaeta.

14. Denzin, N., \& Lincoln, Y. (2012). El campo de la investigación cualitativa en Manual de Investigación Cualitativa. Madrid: Gedisa.

15. Dongre, A. R, Deshmukh, P. R., Kalaiselvan, G., \& Upadhyaya, S. (2009). Application of qualitative methods in health research: An overview. Online Journal of Health Allied Sciencess, 8(4). Recuperado de http://www.ojhas.org/issue32/2009-4-3.htm.

16. Fanjul, C., \& González, C. (2011). La influencia de los modelos somáticos de los anuncios publicitarios en la vigorexia masculina: un estudio experimental en adolescentes. Zer, 16(3), 265-284.

17. Fisher, S. (1986). Development and structure of body image. Hillsdale, New Jersey: Lawrence Erlmaum Associates. Inc., Publishers. 
18. Gómez, A., Sánchez, B., \& Navarrete, M. (2012). Insatisfacción y distorsión de la imagen corporal en adolescentes de doce a diecisiete años de edad. Ágora para la EF y el Deporte, 15, 54 -63.

19. Gómez, G. (2014). Imagen corporal y orientación sexual: deseo de un modelo corporal masculino ideal. México: Editorial Fénix.

20. González, B. (1999). Los estereotipos como factor de socialización en el género. Comunicar, 12, 79-88.

21. Hamui-Sutton., \& Varela-Ruiz, M. (2013). La técnica de grupos focales. Revista en Investigación en Educación Médica, 2(1), 55-60.

22. Chávez-Rosales, E., Camacho, E. J., Maya, M. A., \& Márquez, O. (2012). Conductas alimentarias y sintomatología de trastornos del comportamiento alimentario en estudiantes de nutrición. Revista Mexicana de Trastornos Alimentarios, 3(1), 29-37.

23. Ianotti, C. (2005). Contraste de la percepción de la imagen corporal entre hombres y mujeres. (Tesis de licenciatura inédita). Universidad Abierta Interamericana, Chile. Recuperado de http://imgbiblio.vaneduc.edu.ar/fulltext/files/TC061964.pdf

24. Kerlinger, F., \& Lee, H. (2002). Investigación del comportamiento: métodos de investigación en ciencias sociales. ( $4^{\text {a }}$ ed.) México: McGraw-Hill.

25. Lagos, R., Quilodrán, V., \& Viñuela, N. (2012). Percepción de la imagen corporal de mujeres y hombres de primer año ingreso 2010, en la Universidad Autónoma de Chile, sede Temuco, región de la Araucanía. Revista Digital de Buenos Aires, 17(169). Recuperado de http://www.EFDeportes.com/

26. López, P., Padro, J., Montilla, M., Molina, Z., Da Silva, G., \& Arteaga, F. (2008). Insatisfacción por la imagen corporal y la baja autoestima por la apariencia física en estudiantes de la facultad de medicina de la universidad de los andes del estado merida venezuela. Revista en Ciencias del Movimiento Humano y Salud, 5(19, 1-14.

27. Medina-Mora, M.E., Borges, G., Lara., C., Blanco, J., Fleiz, C., Villatoro, J.A. et al. (2003). Prevalencia de trastornos mentales y uso de servicios: resultados de la Encuesta Nacional de Epidemiología psiquiátrica en México. Salud Mental, 26, 1-16.

28. Mendieta-Izquierdo, G. (2014). Percepción de cuerpo e imagen corporal masculina: una revisión narrativa. Cuadernos Hispanoamericanos de Psicología 14(1), 17-30.

29. Moreno, M., \& Ortiz, G. (2009). Trastorno alimentario y su relación con la imagen corporal y la autoestima en adolescentes. Terapia Psicológica, 27, 181-190.

30. Muñoz, A. (2014). La imagen corporal en la sociedad del siglo XXI. (Tesis de especialidad inédita). Universidad Internacional de 
Catalunya,

España.

Recuperado

de

http://www.recercat.cat/bitstream/handle/2072/242794/Ana_Mar\%C3 $\% \mathrm{ADa} \_\mathrm{Mu} \% \mathrm{C} 3 \% \mathrm{~B} 1 \mathrm{oz} \_\mathrm{L} \% \mathrm{C} 3 \% \mathrm{~B} 3$ pez.pdf?sequence=1

31. Múñoz-Cano, J. M., Córdova-Hernández, J. A., \& Boldo-León, X. M. (2012). Ambiente obesogénico y biomarcadores anómalos en escolares de Tabasco, México. Salud en Tabasco, 18(3), 87-95.

32. Otero, B. (2012). Nutrición. México: Red Tercer Milenio.

33. Philips, J., \& Drummond, M. J. N. (2001). An investigation into the body image perception, body satisfaction and exercise expectations of male fitness leaders: Implications for professional practice. Leisure Studies 20(2), 95-105.

34. Raich, R. M. (2004). Una perspectiva desde la psicología de la salud de la imagen corporal. Avances en Psicología Latinoamericana, 22, $15-27$.

35. Raich, R. M. (2016). La tiranía de la imagen. Barcelona: Singlatana.

36. Raich, R.M., Sánchez-Carracedo, D., \& López, G. (2008). Alimentación, modelo estético femenino y medios de comunicación: Cómo formar alumnos críticos en la educación secundaria. Barcelona, Graó.

37. Salaberria, K., Rodríguez, S., \& Cruz, S. (2007). Percepción de la imagen corporal. Osasunaz, 8, 171-183.

38. Silverman, D. (2016). Qualitative research. Washington, D.C.: Sage.

39. Sociedad Mexicana de Psicología. (2010). Código Ético del Psicólogo. (5a Ed.) México: Trillas.

40. Thompson, J. K., \& Heinberg, L. (1999). The Media's influence on body image disturbance and eating disorders: We've reviled them, now can we rehabilitate them? Journal of Social Issues, 2, 339-353.

41. Thompson, J. K., Schaefer, L. M., \& Menzel, J. E. (2012). Internalization of thin-ideal and muscular-ideal. In Cash, T. F. (Ed.). Encyclopedia of body image and human appearance (pp. 499-504). San Diego: Academic Press.

42. Thompson, J. K., \& Smolak, L. (2001). Body image, eating disorders, and obesity in youth: Assessment, prevention, and treatment. Washington, DC, EUA: American Psychological Association.

43. Tiggemann, M., Martins, Y., \& Churchett, L. (2008). Beyond muscles: Unexplored parts of men's body image. Journal of Health Psychology, 13(8), 1163-1172.

44. Unikel, C., Díaz de León, C., \& Rivera, J. A. (2016). Conductas alimentarias de riesgo y correlatos psicosociales en estudiantes universitarios de primer ingreso con sobrepeso y obesidad. Salud Mental, 39(39, 11-22. 
45. Unikel, C. Villatoro, J. A., Medina-Mora, M. E., Fleiz, C., Alcántar, E. N., \& Hernández, S. A. (2000). Coductas alimentarias de riesgo en adolesentes mexicanos. Datos de población estudiatil del Distrito Federal. La Revista de Investigación Clínica, 52(2), 140-148.

46. Unikel-Santoncini, C., Bojórquez-Chapela, L., Villatoro-Velázquez, J., Fleiz-Bautista, C., \& Medina-Mora, M. E. (2006). Conductas alimentarias de riesgo en población estudiantil del Distrito Federal: tendencias 1997-2003. La Revista de Investigación Clínica, 58(1), 15-27.

47. Vaquero-Cristobal, R., Alacid, F., Muyor, J., \& López-Minarro, P. (2013). Imagen corporal: revisión bibliográfica. Nutrición Hospitalaria, 28, 60-73. 